\title{
Origin of The Failure of Density Functional Theories in Predicting Inverted Singlet-Triplet Gaps
}

\author{
Soumen Ghosh*, Kalishankar Bhattacharyya
}

Max-Planck-Institut für Kohlenforschung, D45470 Mülheim an der Ruhr, Germany

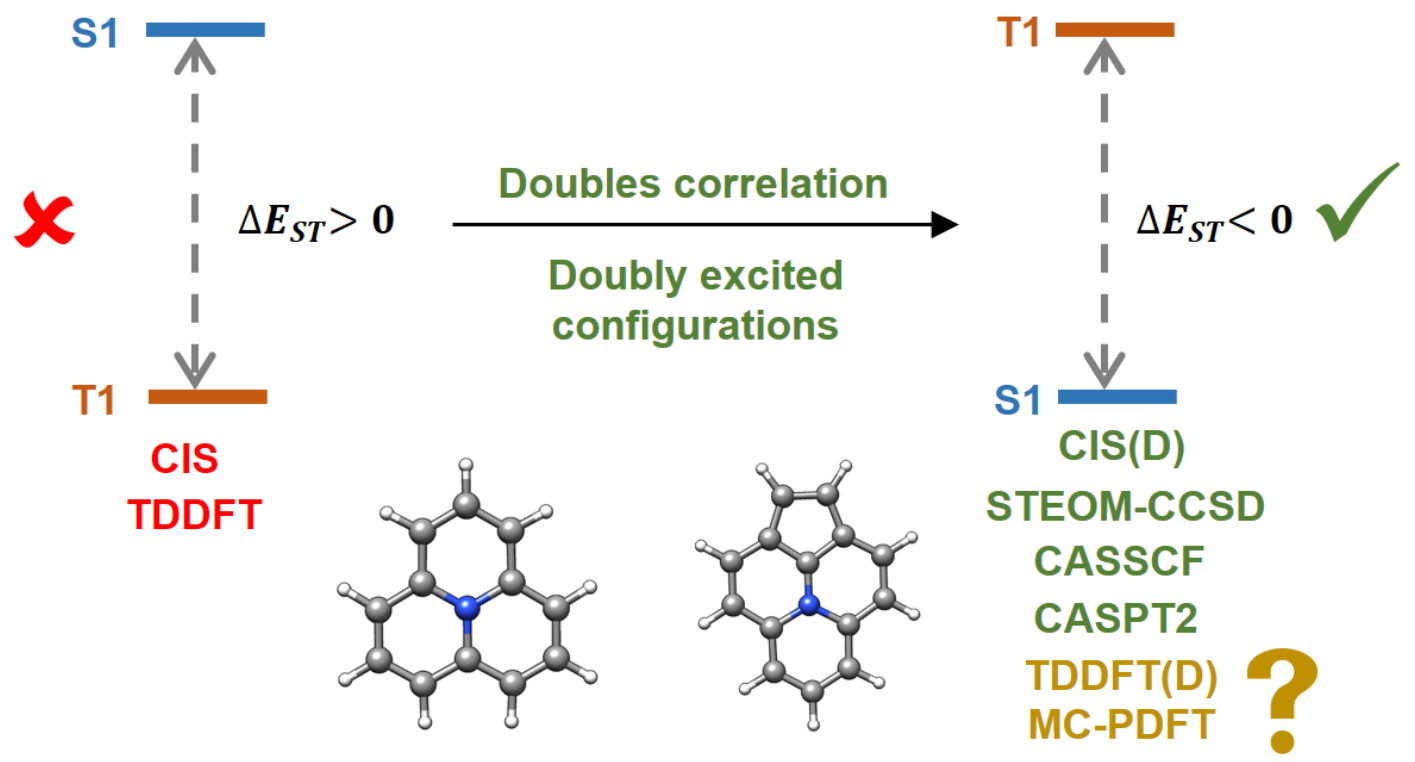

ABSTRACT:

Recent experimental and theoretical studies have shown several new organic molecules that violate Hund's rule and have the first singlet excited state lower in energy than the first triplet excited state. While many correlated single reference wave function methods have successfully predicted excited state energetics of these low-lying states, linear-response time-dependent density functional theory (LR-TDDFT) fails to predict the correct excited state energy ordering. Herein, we have shown that it is possible to get inverted singlet-triplet gaps within the density functional theory framework by going beyond the singly excited state framework of conventional LRTDDFT. Going beyond Kohn-Sham density functional theory (KS-DFT), we have demonstrated that a combined wave function and density functional method resulting in amulticonfiguration 
pair-density functional theory (MC-PDFT), in some cases, can predict inverted singlet-triplet gaps. Consequently, we have identified that both the missing doubly excited configurations and the form of the exchange-correlation functionals are the foremost grounds for the failure of the LR-TDDFT method. We have also compared the accuracy of single reference correlated wave function methods for these low-lying singlet and triplet excited states to multireference second-order perturbation theory.

\section{INTRODUCTION:}

Ground state electronic structures and low-lying singlet and triplet excited states play important roles in organic electronic materials. ${ }^{1,2,3,4,5,6}$ In organic light-emitting diodes (OLEDs), charge recombination is an important step that produces singlet or triplet excitons from spatially separated holes and electrons. Generation of photons from singlet excitons happen through a spin-allowed de-excitation process. However, due to the spin forbidden nature of the de-excitation process of triplet excitons, these often contribute to energy loss. Several strategies have been developed for transforming triplet excitons produced during the recombination process to singlet excitons. In many OLEDs, triplet state $\left(T_{1}\right)$ to singlet state $\left(S_{1}\right)$ reverse intersystem crossing (RISC) is achieved by tuning the energy gap ( $\left.\triangle \mathrm{E}_{\mathrm{ST}}\right)$ gap between energetically close $S_{1}$ and $T_{1}$ excited states. The process of RISC followed by de-excitation is referred to as thermally activated delayed fluorescence (TADF) ${ }^{7,9}$ Both theoretical and computational modeling has contributed significantly in finding efficient design principles for TADF materials. ${ }^{10,11,12,13}$ Most materials developed so far have small but positive $\Delta \mathrm{E}_{\mathrm{ST}}$ gaps as Hund's rule ${ }^{14}$ predicts that the first excited state of a closed-shell molecule is a $T_{1}$ state and the $S_{1}$ excited state will always be higher in energy. However, some N-doped triangulate molecules have been shown to violate Hund's rule by having inverted singlet-triplet gaps $\left(\Delta \mathrm{E}_{S T}<0\right) .^{15,16}$ These materials benefit from the efficient reverse 
intersystem crossing process from $T_{1}$ to $S_{1}$ state, leading to substantial fluorescence rates, thus, in turn, suitable candidates for TADF materials.

In recent publications, computational studies have proposed several new molecules that can produce inverted singlet-triplet gaps $\left(\Delta \mathrm{E}_{S \mathrm{ST}}<0\right) .{ }^{10-13}$ Pollice et al identified computationally a set of organic chromophores that showed efficient TADF processes using equation-of-motion coupled-cluster singles and doubles (EOM-CCSD) ${ }^{17}$ as the method of choice for large-scale screening. ${ }^{11}$ Sancho-Garcia and co-workers have suggested that small chemical modifications of the triangle derivatives can produce good candidates for inverted singlet-triplet gap using several correlated wave function methods. ${ }^{13}$ While many wave function-based electronic structure methods, such as configuration interaction singles with doubles correction $(\mathrm{CIS}(\mathrm{D}))^{18}$, EOM$\mathrm{CCSD}^{17}$ and similarity transformed equation-of-motion coupled-cluster singles and doubles (STEOM-CCSD) ${ }^{19,20}$, have correctly predicted inverted singlet-triplet gaps, one of the most widely used excited-state electronic structure method, linear response time-dependent density functional theory (LR-TDDFT) $)^{21}$ has failed to predict the inversion of singlet-triplet gaps. Computational studies, in the past, pointed towards the inability of LR-TDDFT to incorporate double excitations by going beyond adiabatic approximation ${ }^{22}$ as the source of this error. ${ }^{10}$ However, most of the studies have been limited within the framework of conventional LR-TDDFT methods, and the importance of doubles excitations in predicting inverted singlet-triplet gaps have not been investigated yet. In this article, we have investigated if the inclusion of correlation obtained from double excitations to the conventional LR-TDDFT calculations can predict inverted singlet-triplet gaps. Going beyond conventional KS-DFT methods, we have examined the accuracy of a combined wave function and density functional method, known as multiconfiguration pair-density functional theory (MC-PDFT) ${ }^{23,24}$, developed by Li Manni et al., in predicting energetics of low- 
lying singlet and triplet excited states of the test systems. Finally, we have examined the accuracy of single reference correlated wave function methods with respect to multireference second-order perturbation theory (MRPT2).

\section{RESULTS AND DISCUSSION:}
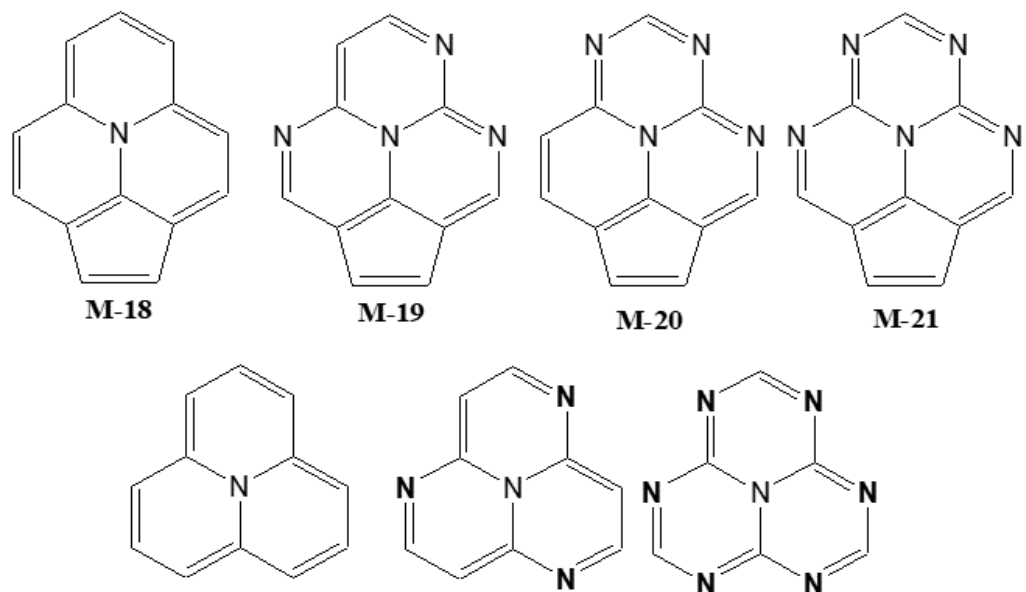

Azine
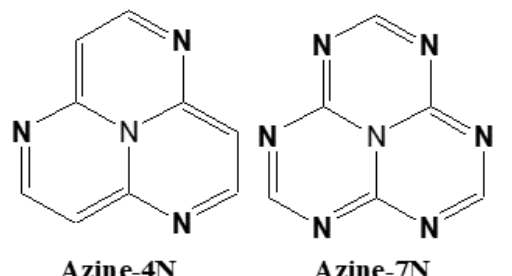

Figure 1. Chemical structures of molecules investigated in this work.

Within the LR-TDDFT regime, excitation energies can be calculated from the solution of the non-Hermitian eigenvalue problem:

$$
\left[\begin{array}{cc}
A & B \\
B^{*} & A^{*}
\end{array}\right]\left[\begin{array}{l}
X \\
Y
\end{array}\right]=\Omega_{T D D F T}\left[\begin{array}{cc}
1 & 0 \\
0 & -1
\end{array}\right]\left[\begin{array}{l}
X \\
Y
\end{array}\right]
$$

where $X$ and $Y$ are excitation and de-excitation amplitudes, respectively and $\Omega_{T D D F T}$ is the excitation energy matrix. Matrix elements corresponding to $A$ and $B$ matrices for a general hybrid exchange-correlation functional can be expressed as:

$$
\begin{gathered}
A_{i a, j b}=\delta_{i j} \delta_{a b}\left(\varepsilon_{a}-\varepsilon_{b}\right)+(i a \mid j b)-C_{H F}(i j \mid a b)+\left(1-C_{H F}\right)\left(i a\left|\hat{f}_{x c}\right| j b\right) \\
B_{i a, j b}=(i a \mid b j)-C_{H F}(i b \mid a j)+\left(1-C_{H F}\right)\left(i a\left|\hat{f}_{x c}\right| b j\right)
\end{gathered}
$$


where $C_{H F}$ is the percentage of HF exchange in the exchange-correlation functional, $\hat{f}_{x c}$ is the exchange-correlation kernel, $\varepsilon$ is the energy eigenvalues. In the case of RPA or TDHF, $C_{H F}$ is equal to 1 and the exchange-correlation kernel is zero. Head-Gordon and coworkers developed CIS(D) method to account for correlation energy resulting from double excitations in a perturbative way. ${ }^{18}$

Neese and Grimme extended this approach to LR-TDDFT for double hybrid exchange-correlation functionals following the same approach as CIS(D). ${ }^{25}$ Excitation energies in the doubles corrected TDDFT method,

$$
\Omega_{T D D F T(D)}=\Omega_{T D D F T}+a_{c} \Delta_{(D)}
$$

where $\Delta_{(D)}$ is the perturbative doubles correction obtained from MP2 and $a_{c}$ is the scaling factor for MP2 correlation in double hybrid functionals. Scaling factor, $a_{c}$, is equal to 1 for CIS(D) method.

All the methods mentioned above, including coupled-cluster methods like EOM-CCSD and STEOM-CCSD, work only when the excited state is a predominately single excited state in nature. Herein, we have examined electronic structures of ground and first excited $\mathrm{S}_{1}$ and $\mathrm{T}_{1}$ states of seven molecules as shown in Figure 1, which are predicted to have inverted or isoenergetic $\mathrm{S}_{1}$ and $\mathrm{T}_{1}$ states by previous theoretical and experimental studies. ${ }^{11,20}$ As shown in Figure 2, both CIS and LR-TDDFT methods that only consider the single excitation space predict positive singlet-triplet gaps for all these molecules. Interestingly, all types of exchange-correlation functionals demonstrate positive singlet-triplet gaps $\left(\Delta \mathrm{E}_{\mathrm{ST}}>0\right)$ with the conventional LR-TDDFT method (see the table S1 in the SI). In Figure 2, we have shown CAM-B3LYP as a representative example of the performance of exchange-correlation functionals within LR-TDDFT regime. 
Within a single reference framework, previous studies have shown that, unlike KS-DFT, correlated wave function methods have successfully predicted the inversion of singlet-triplet gaps for these types of molecules. In figure 2, we have shown singlet-triplet gaps of all seven molecules with CIS, CIS(D), and domain-based local pair natural orbital (DLPNO) STEOM-CCSD methods. Like LR-TDDFT, CIS can not obtain negative singlet-triplet gaps for all seven molecules (Figure 2). CIS(D) and DLPNO-STEOM-CCSD, on the other hand, successfully predict this behavior for all seven molecules (Figure 2). The difference between CIS and CIS(D) clearly shows that correlation due to the double excitations needs to be accounted for during the calculation to be able to get inverted singlet-triplet gaps. The success of the higher-level correlated methods like DLPNO-STEOM-CCSD in predicting this property can be attributed to correlation originating from higher-order excitations. The discrepancy between the performance of CIS and CIS(D) method, indicates that the failure of conventional LR-TDDFT to take double excitations or even correlation resulting from double excitations into account can be a major reason for its failure.

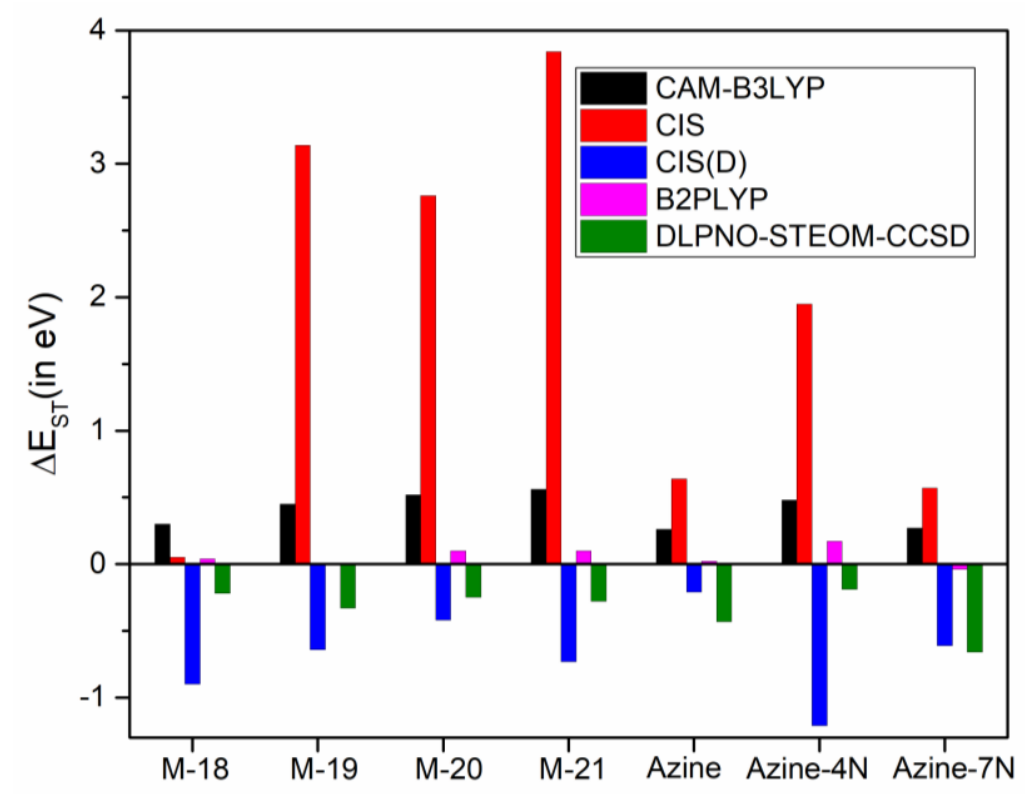


Figure 2. Single-triplet gaps ( $\left.\Delta \mathrm{E}_{\mathrm{ST}}\right)$ for all seven chromophores were computed with different electronic structure methods using def2-TZVP basis sets.

It is possible to recover higher-order correlation in the KS-DFT framework with the use of double-hybrid functionals. In the same philosophy of CIS(D), one can introduce a double excitation correlation in the LR-TDDFT method. However, such a method is only consistent with the concept of double-hybrid functionals as introduced by Neese and Grimme. ${ }^{19}$ In the doubles corrected LR-TDDFT (LR-TDDFT(D)) approach, double correction is scaled according to the percentage of non-local correlation (MP2 correlation in the case of B2PLYP) present in the exchange-correlation functional for the ground state. As shown in Figure 2, B2PLYP produced mixed results as it has predicted negative singlet-triplet gaps for Azine-7N and M19 but predicted positive singlet-triplet gaps for the remaining molecules. CIS(D) and LR-TDDFT(D) used for double hybrid functionals differ by the scaling factor for the exact exchange integral introduced by the functional form (equation 4 ) and also by the exchange-correlation kernel $\left(\hat{f}_{x c}\right)$ (equations 2 and 3). The nonlocal correlation part in B2PLYP (i.e., MP2 contribution) is scaled according to the functional form (equation 4). CIS(D) predicts negative singlet-triplet gaps for all of the test cases, while B2PLYP only predicts negative singlet-triplet gaps for two systems. Clearly, it renders that the origin of this difference lies in the functional form of B2PLYP. However, these results are truly encouraging as many previous studies concluded that it is not possible for conventional exchange-correlation functionals within adiabatic approximation to predict negative singlet-triplet gaps for such systems but our study has shown otherwise. Efforts should be made in the future to develop new functionals, specifically double-hybrid functionals, that are better suited for application in this type of systems. 
All of the single reference methods studied here, despite their success, only applicable for excited states that are dominated by single excitations. However, considering the important role that double excitations play in the singlet-triplet energetics of these molecules, the ideal method should treat both single and double excited states with equal accuracy. Figure 3 shows the percentage of doubly excited configuration in the first singlet excited state from restricted active space self-consistent field (RASSCF) calculations. Our analysis shows that S1 state for all seven molecules have significant double excitation contribution even though the $\mathrm{S} 1$ excited states are still predominately single excited states.

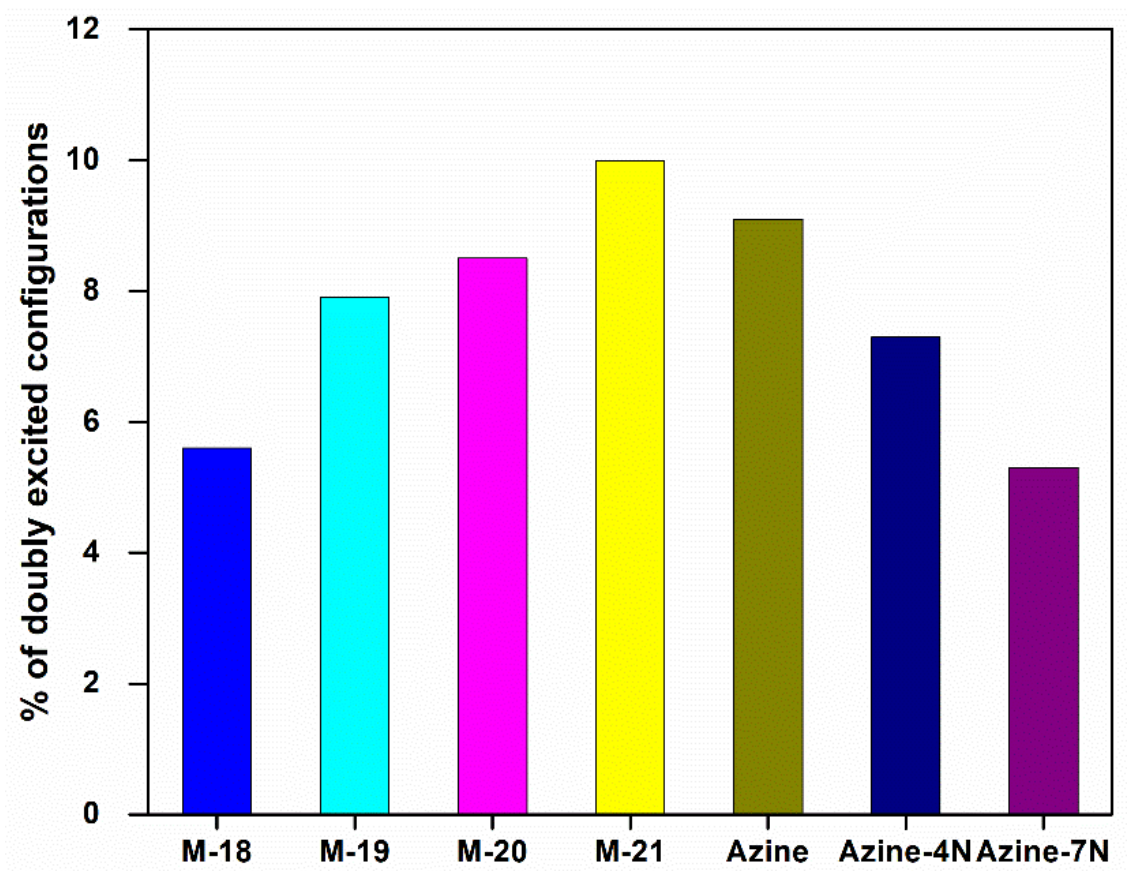

Figure 3. Percentage (\%) of doubly excited configurations in the RASSCF wave function the S1 state of all the chromophores in Figure 1. 
Table 1. Vertical $\mathrm{S}_{0}-\mathrm{S}_{1}$, and $\mathrm{S}_{0}-\mathrm{T}_{1}$ excitation energies (in $\mathrm{eV}$ ) of the studied chromophores based on double hybrid functionals. Def2-TZVP basis is employed for all calculations.

\begin{tabular}{cccccccccccc} 
& \multicolumn{3}{c}{ RASSCF } & \multicolumn{3}{c}{ RASPT2 } & \multicolumn{3}{c|}{ tPBE } \\
& \multicolumn{1}{c}{ S1 } & T1 & $\Delta$ EST & S1 & T1 & $\Delta E_{S T}$ & S1 & T1 & $\Delta E_{S T}$ \\
M18 & 2.03 & 2.27 & -0.24 & 1.98 & 1.92 & 0.06 & 2.29 & 2.12 & 0.17 \\
M19 & 2.51 & 2.61 & -0.10 & 2.56 & 2.62 & -0.06 & 2.91 & 2.96 & -0.05 \\
M20 & 2.35 & 2.59 & -0.24 & 2.52 & 2.58 & -0.06 & 2.97 & 2.83 & 0.14 \\
M21 & 2.71 & 2.86 & -0.15 & 2.76 & 2.92 & -0.16 & 3.08 & 3.22 & -0.14 \\
Azine & 0.65 & 0.93 & -0.28 & 0.86 & 0.89 & -0.03 & 1.37 & 1.2 & 0.17 \\
\hline Azine-4N & 2.24 & 2.22 & 0.02 & 1.89 & 1.84 & 0.05 & 2.03 & 1.94 & 0.09 \\
\hline Azine-7N & 2.56 & 2.95 & -0.39 & 2.54 & 2.67 & -0.13 & 2.8 & 2.69 & 0.11 \\
\hline
\end{tabular}

Multireference second-order perturbation theory (MRPT2) ${ }^{26}$ can treat both singly and doubly excited states with similar accuracy. MC-PDFT can be another method of choice that properly takes into account doubly excited configurations but within the density functional theory framework. Both MC-PDFT and MRPT2 methods are based on wave functions obtained from the multiconfigurational self-consistent field (MCSCF) calculations. While MRPT2 recovers dynamical correlation using second-order perturbation theory, MC-PDFT recovers dynamical correlation using a new type of exchange-correlation functionals called on-top pair-density functionals. Total MC-PDFT energy of $\Psi_{M C S C F}$ state,

$$
E^{M C-P D F T}=V_{n n}+\left\langle\Psi_{M C S C F}\left|T+V_{n e}\right| \Psi_{M C S C F}\right\rangle+V_{C}[\rho]+E_{o t}[\rho, \Pi]
$$


where $V_{n n}$ is nuclear repulsion energy, $\left\langle\Psi_{M C S C F}\left|T+V_{n e}\right| \Psi_{M C S C F}\right\rangle$ is the summation of kinetic and nuclear-electron attraction energies obtained from MCSCF calculations, $V_{C}[\rho]$ is the classical electron-electron repulsion energy and $E_{o t}[\rho, \Pi]$ is the on-top pair-density functional.

In our calculations, we have used a variation of the MCSCF method, restricted active space self-consistent field (RASSCF) method and the corresponding PT2 method, RASPT2, for studying the excited state of these systems. RASPT2 predicted singlet-triplet gaps of all other molecules to be either negative or close to zero. Interestingly, the on-top density functionals only predicted negative singlet-triplet gaps for M19 and M21 (see table 1 and table S3), even though MC-PDFT uses the same reference wave function as RASPT2. First two terms in the MC-PDFT energy expression is same as MCSCF and the classical electron-electron repulsion term is exact. So, the failure of MC-PDFT can be traced back to the functional form of pair-density functionals (tPBE, ftPBE, tBLYP and ftBLYP). Similar to KS-DFT, better functional form needs to be developed in the framework of MC-PDFT for accurate treatment of this type of excited state energetics. Interestingly, even though both B2PLYP and pair-density functionals show mixed successes for this set of molecules, these two methods treat double excitation contributions in a very different way. While doubles corrected LR-TDDFT using B2PLYP functional takes into account only the correlation contributions coming from double excitations but it does not include doubly excited configurations in the excited state wave functions. On the other hand, MC-PDFT uses MCSCF wave function that includes doubly excited configurations. In spite of this fundamental difference, both these approaches are promising for this type of problems. Interestingly, even though results are quantitatively different between single reference methods like CIS(D) and DLPNO-STEOMCCSD and multireference RASPT2 method, they produced similar qualitative trends.

\section{CONCLUSION:}


In this article, we have shown that the inability of conventional LR-TDDFT to take double excitation contributions into account as one of the major reasons for the failure of KS-DFT in predicting singlet-triple inversion. It is possible to obtain negative singlet-triplet gaps by using exchange-correlation functionals with non-local correlation (i.e., double-hybrid functionals) vis$\grave{a}$-vis combined wave function and density functional theory methods, such as MC-PDFT. However, the performance of both approaches is not consistent for all molecules in our test set. New functionals need to be developed for both of these theories that can model singlet-triplet energetics in similar systems. Within KS-DFT and MC-PDFT framework, it is important to choose a proper functional form and excited-state methods for studying excited state energetics of this type of system. We have also pointed out that MRPT2 and single reference wave function methods that account for correlations originating from double excitations produce qualitatively similar results.

\section{COMPUTATIONAL DETAILS:}

All KS-DFT, LR-TDDFT, CIS, CIS(D) and DLPNO-STEOM-CCSD calculations were performed using a development version of the ORCA suits of programs based on version 4.2. ${ }^{27}$ All RASSCF, RASPT2 and MC-PDFT calculations were performed using OpenMolcas software package ${ }^{28}$ (v19.11, tag 1689-g1367d6fd9). All RASPT2 calculations used an imaginary shift of $5.44 \mathrm{eV}$ to alleviate intruder states. ${ }^{29}$ All calculations used Alrich's def 2 triple zeta with polarization basis functions, def2-TZVP ${ }^{30}$. RASSCF calculation are performed for all seven molecule using full $\pi$ valence active spaces. For RASSCF calculations on Azine, Azine-4N and Azine-7N, RAS2 only includes HOMO and LUMO, RAS1 includes 6 occupied orbitals, RAS3 includes 6 unoccupied orbitals and maximum 2 holes and 2 electrons are allowed in RAS1 and RAS3, respectively. For RASSCF calculations on M-18, M-19, M-20 and M-21, RAS2 includes HOMO, HOMO-1, LUMO 
and LUMO+1, RAS1 includes 6 occupied orbitals, RAS3 includes 6 unoccupied orbitals and maximum 2 holes and 2 electrons are allowed in RAS1 and RAS3, respectively. Geometries of molecules in the test set were optimized in the gas-phase at B3LYP $31 /$ def2-TZVP level of theory, including Grimme’s D3 dispersion correction ${ }^{32}$ and Becke-Johnson damping function ${ }^{33}$.

\section{ASSOCIATED CONTENT}

\section{Supporting Information}

Full computational details, coordinates of the optimized structures.

\section{AUTHOR INFORMATION}

\section{Corresponding Author}

Corresponding Author: (S. G.) Email:chemsghosh@gmail.com

\section{Notes}

The authors declare no competing financial interests.

SG and KB contributed equally to this work.

\section{ACKNOWLEDGMENT}

The authors thank Dr. Varinia Bernales and Dr. Pragya Verma for helpful suggestions and discussions. The authors also acknowledge generous support from the Alexander von Humboldt foundation.

\section{REFERENCES}


${ }^{1}$. Nakano, M. in Excitation Energies and Properties of Open-Shell Singlet Molecules Applications to a New Class of Molecules for Nonlinear Optics and Singlet Fission, ed. G. Maroulis, Springer, Heidelberg, 2014.

2. Feringa, B. L.; Delden, R. A. V.; Koumura, N.; Geertsema, E. M. Binaphthalene Molecules with Tetrathiafulvalene Units: CD Spectrum Modulation and New Chiral Molecular Switches by Reversible Oxidation and Reduction of Tetrathiafulvalene Units. Chem. Rev. 2000, 100, 17891816.

${ }^{3}$. Sato, K.; Shizu, K.; Yoshimura, K.; Kawada, A.; Miyazaki, H.; Adachi, C. Organic luminescent molecule with energetically equivalent singlet and triplet excited states for organic light-emitting diodes. Phys. Rev. Lett. 2013, 110, 247401.

${ }^{4}$. Nobuyasu, R. S.; Ren, Z.; Griffiths, G. C.; Batsanov, A. S.; Data, P.; Yan, S.; Monkman, A. P.; Bryce, M. R.; Dias, F. B. Rational Design of TADF Polymers Using a Donor-Acceptor Monomer with Enhanced TADF Efficiency Induced by the Energy Alignment of Charge Transfer and Local Triplet Excited States. Adv. Opt. Mater. 2016, 4, 597-607.

5. Dias, F. B.; Santos, J.; Graves, D. R.; Data, P.; Nobuyasu, R. S.; Fox, M. A.; Batsanov, A. S.; Palmeira, T.; Berberan-Santos, M. N.; Bryce, M. R.; et al. The role of local triplet excited states and D-A relative orientation in thermally activated delayed fluorescence: Photophysics and devices. Advanced Science 2016, 3, 1600080.

6. Samanta, P. K.; Kim, D.; Coropceanu, V.; Brédas, J. L. Up-Conversion Intersystem Crossing Rates in Organic Emitters for Thermally Activated Delayed Fluorescence: Impact of the Nature of Singlet vs Triplet Excited States. J. Am. Chem. Soc. 2017, 139, 4042-4051. 
7. Endo, A.; Sato, K.;Yoshimura, K.; Kai, T.; Kawada, A.; Miyazaki, H.; Adachi, C. Efficient upconversion of triplet excitons into a singlet state and its application for organic light emitting diodes. Appl. Phys. Lett. 2011, 98, 083302.

${ }^{8}$. Uoyama, H.; Goushi, K.; Shizu, K.; Nomura, H.; Adachi, C. Highly efficient organic lightemitting diodes from delayed fluorescence. Nature 2012, 492, 234-238.

9. Liu, Y.; Li, C.; Ren, Z.; Yan, S.; Bryce, M. R. All-organic thermally activated delayed fluorescence materials for organic light-emitting diodes. Nat. Rev. Mater. 2018, 3, 18020.

${ }^{10}$. de Silva, P. Inverted Singlet-Triplet Gaps and Their Relevance to Thermally

Activated Delayed Fluorescence. J. Phys. Chem. Lett. 2019, 10, 5674-5679.

11. Pollice, R.; Friederich, P.; Lavigne, C.; dos Passos Gomes, G.; Aspuru-Guzik*, A. Organic Molecules with Inverted Gaps between First Excited Singlet and Triplet States and Appreciable Fluorescence Rates. Matter 2021, 4, 1654-1682.

12. Pios, S.; Huang, X.; Sobolewski, A. L.; Domcke, W. Triangular boron carbon nitrides: An unexplored family of chromophores with unique properties for photocatalysis and ptoelectronics. Phys. Chem. Chem. Phys., 2021, DOI: 10.1039/D1CP02026A.

13. Ricci, G.; San-Fabián, E.; Olivier, Y.; Sancho-García, J. C. Singlet-Triplet Excited-State Inversion in Heptazine and Related Molecules: Assessment of TD-DFT and ab initio Methods. Chem. Phys. Chem. 2021, 22, 553-560.

${ }^{14}$. Hund, F. Z. Zur deutung verwickelter spektren, insbesondere der elemente scandium bis nickel. Physik 1925, 33, 345-371.

${ }^{15}$. Leupin, W.; Wirz, J. Low-Lying Electronically Excited States of Cycl[3.3.3]azine, a Bridged

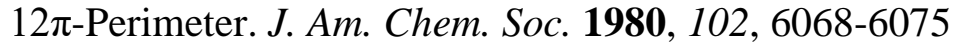

${ }^{16}$. Miyajima, D.; Aizawa, N.; Pu, Y.; Nihonyanagi, A.; Ibuka, R.; Inuzuka, H.; Dhara, B.; Koyama, Y.; Araoka, F. Delayed Fluorescence from Inverted Singlet and Triplet Excited States for Efficient Organic Light-Emitting Diodes. 2021, Preprint available at Research square DOI https://doi.org/10.21203/rs.3.rs-478258/v1 . 
17. Head-Gordon, M.; Rico, R. J.; Oumi, M.; Lee, T. J. A doubles correction to electronic excited states from configuration interaction in the space of single substitutions. Chem. Phys. Lett. 1994, 219, 21-29.

${ }^{18}$. Rhee, Y. M.; Head-Gordon, M. Scaled Second-Order Perturbation Corrections to Configuration Interaction Singles: Efficient and Reliable Excitation Energy Methods. J. Phys. Chem. A 2007, $111,5314-5326$.

${ }^{19}$. Nooijen, M.; Bartlett, R. J. Similarity transformed equation-of-motion coupled-cluster theory: Details, examples, and comparisons. J. Chem. Phys. 1997, 107, 6812-6830.

${ }^{20}$. Bhattacharyya, K. Can TDDFT Render the Electronic Excited States Ordering of Azine Derivative? A Closer Investigation with DLPNO-STEOM-CCSD. Chem. Phys. Lett. 2021, 779, 138827.

${ }^{21}$. Runge, E.; Gross, E. K. U. Density-Functional Theory for Time Dependent Systems. Phys. Rev.

Lett. 1984, 52, 997-1000.

${ }^{22}$. Elliott, P.; Goldson, S.; Canahui, C.; Maitra, N. T. Perspectives on double-excitations in TDDFT. Chem. Phys. 2011, 391, 110-119.

23. Li Manni, G.; Carlson, R. K.; Luo, S.; Ma, D.; Olsen, J.; Truhlar, D. G.; Gagliardi, L. Multi-

Configuration Pair-Density Functional Theory. J. Chem. Theory Comput. 2014, 10, 3669-3690.

${ }^{24}$. Ghosh, S.; Verma, P.; Cramer, C. J.; Gagliardi, L.; Truhlar, D. G. Combining Wave Function Methods with Density Functional Theory for Excited States. Chem. Rev. 2018, 118, 7249-7292.

25. Grimme, S.; Neese, F. Double-Hybrid Density Functional Theory for Excited Electronic States of Molecules. J. Chem. Phys. 2007, 127, 154116.

${ }^{26}$. Hirao, K. Multireference Møller-Plesset method. Chem. Phys. Lett. 1992, 190, 374-380

27. Neese, F. Software Update: The Orca Program System, Version 4.0. WIREs Comput. Mol. Sci. 2018, 8, No. e1327.

${ }^{28}$. Fdez Galvan, I.; Vacher, M.; Alavi, A.; Angeli, C.; Aquilante, F.; Autschbach, J.; Bao, J. J.; Bokarev, S. I.; Bogdanov, N. A.; Carlson, R. K.; Chibotaru, L. F.; Creutzberg, J.; Dattani, N.; Delcey, M. G.; Dong, S. S.; Dreuw, A.; Freitag, L.; Frutos, L. M.; Gagliardi, L.; Gendron, F.; Giussani, A.; Gonzalez, L.; Grell, G.; Guo, M.; Hoyer, C. E.; Johansson, M.; Keller, S.; Knecht, S.; Kovacevic, G.; Kallman, E.; Li Manni, G.; Lundberg, M.; Ma, Y.; Mai, S.; Malhado, J. P.; 
Malmqvist, P. A.; Marquetand, P.; Mewes, S. A.; Norell, J.; Olivucci, M.; Oppel, M.; Phung, Q. M.; Pierloot, K.; Plasser, F.; Reiher, M.; Sand, A. M.; Schapiro, I.; Sharma, P.; Stein, C. J.; Sørensen, L. K.; Truhlar, D. G.; Ugandi, M.; Ungur, L.; Valentini, A.; Vancoillie, S.; Veryazov, V.; Weser, O.; Wesołowski, T. A.; Widmark, P.-O.; Wouters, S.; Zech, A.; Zobel, J. P.; Lindh, R. OpenMolcas: From Source Code to Insight. J. Chem. Theory Comput. 2019, 15, 5925-5964. ${ }^{29}$.Forsberg, N.; Malmqvist, P. A. Multiconfiguration perturbation theory with imaginary level shift. Chem. Phys. Lett. 1997, 274, 196-204

${ }^{30}$. Weigend, F.; Ahlrichs, R. Balanced basis sets of split valence, triple zeta valence and quadruple zeta valence quality for $\mathrm{H}$ to $\mathrm{Rn}$ : Design and assessment of accuracy. Phys. Chem. Chem. Phys. 2005, 7, 3297-3305.

${ }^{31}$. Becke, A. D. Density-functional thermochemistry. III. The role of exact exchange. J. Chem. Phys. 1993, 98, 5648-5652.

32. Grimme, S.; Antony, J.; Ehrlich, S.; Krieg, H. A consistent and accurate $a b$ initio parametrization of density functional dispersion correction (DFT-D) for the 94 elements $\mathrm{H}-$ Pu. J. Chem. Phys. 2010, 132, 154104.

${ }^{33}$. Chai, J. D.; Head-Gordon, M. Long-range corrected hybrid density functionals with damped atom-atom dispersion corrections. Phys. Chem. Chem. Phys. 2008, 10, 6615-6620. 